\title{
Atomic Resolution Images Using Forbidden Reflections on (111) Face Centered Cubic Nanoparticles
}

\author{
S.-C. Y. Tsen*, P. A. Crozier* and M. Gajdardziska-Josifovska** \\ *Center for Solid State Science, Arizona State University, Tempe, AZ 85287-1704 \\ **Department of Physics, University of Wisconsin Milwaukee, Milwaukee WI 53201
}

We have observed images of the [111] projection of Au nanoparticles $(15 \sim 30 \AA)$ which show strong fringe spacings corresponding to "forbidden" reflections. Cherns first utilized the so-called "forbidden reflections" from evaporated (111) gold films to study atomic steps.[1] Computer modeling of high-resolution transmission electron microscope (HREM) image of the (001) Au film surface has been reported by Krakow.[2] The forbidden reflections are visible only when the thickness of the crystal (which has ..ABCABC.. stacking in the [111] direction) includes a nonintegral number of unit cells. In order to understand the effect of these reflections on the highresolution image of small particles, image simulations on both particle and thin film models of $\mathrm{Au}$ have been undertaken.

Au samples with particle sizes about $60 \AA$ to $100 \AA$ were evaporated directly on to carbon grids. HREM images were recorded using a JEM 4000EX electron microscope operating at $400 \mathrm{kV}$ with resolution about $1.7 \AA$. Figure 1 shows two examples of atomic resolution images of [111] Au particles with their diffractograms. The diffractograms showed 6 strong reflections with the corresponding fringe spacing as large as $2.5 \AA\left(\mathrm{d}_{111} \sim 2.36 \AA\right)$ and the relative angles are $60^{\circ}$. The first allowed bulk reflection from [111] $\mathrm{Au}$ is the (220) corresponding to a lattice spacing of $1.44 \AA$ and is beyond the transfer limit of the microscope. The observed reflections can be indexed as $1 / 3\{422\}$ in the reciprocal space and correspond to the forbidden reflections described by Cherns.

Au thin film models were built with CERIUS Molecular Modeling Program and the HREM images simulation performed with the multi-slice method. Figure 2(a) and 2(b) show the simulated images and the corresponding diffraction patterns for samples of 1,2 and 3 atomic layers thick. For one and two atomic layers there are very strong forbidden reflection and the corresponding HREM images show high contrast for this spatial frequency. One interesting result is the contrast reversal that occurs upon going from one to two layers of atoms. This difference can also be seen in experimental results by comparing Figure 1 (a) and (b). The black dots correspond to the surface atom positions if one extra layer is on the surface. Similar results are obtained if the extra layer is located in the bulk of the crystal (i.e. a stacking fault). Our studies show that models with equal number of layers of A, $\mathrm{B}$ and $\mathrm{C}$ atomic sites have a very weak contrast in their images because the objective lens filters out all the bulk reflections (which are below $1.7 \AA$ for this projection). Models with a non-equal number of layers of $\mathrm{A}, \mathrm{B}$ or $\mathrm{C}$ atomic sites showed strong fringe contrast. The images are similar to the images of hexagonal crystals along [0001] projection and can easily be mistaken.

Nanoparticle models were built with Rhodius program.[3] Figure 3(a) shows the top view of a 3-D projection of a cuboctahedron $\mathrm{Au}$ particle ( 30 $\AA$ and 13 atomic layers) with the [111] direction running along the $\mathrm{c}$ direction of the super cell. The image simulated at $-320 \AA$ defocus and its diffractogram are shown in Figure 3(b) and 3(c). The periodicities are similar to that of the thin film case. The different appearance of the images along [111] projection can be used to identify particles 
with one, two extra atomic layers or with complete $\mathrm{ABC}$ stacking sequence if there are no stacking faults involved.

References:

[1] D. Cherns, Phil. Mag. 30 (1974) 549.

[2] W. Krakow, Ultramicroscopy 4 (1979) 55.

[3] F. J. Botana et al; Proc. 13 ${ }^{\text {th }}$ Int. Cong. on Electron Microsc. $2 b$ (1994) 1085.

$1 \mathbf{a}$
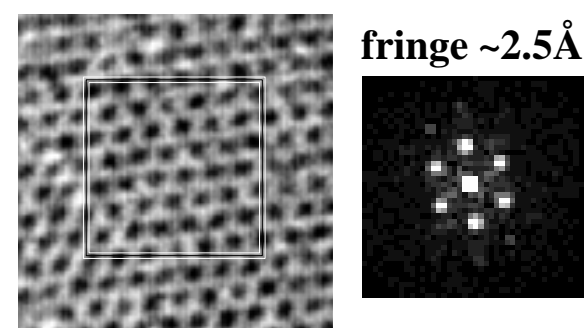

$1 \mathbf{b}$

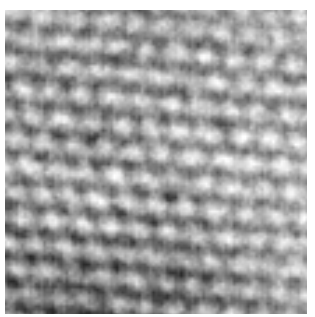

fringe $\sim 2.5 \AA$

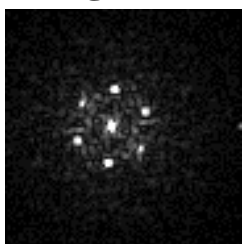

$\mathbf{2 a}$

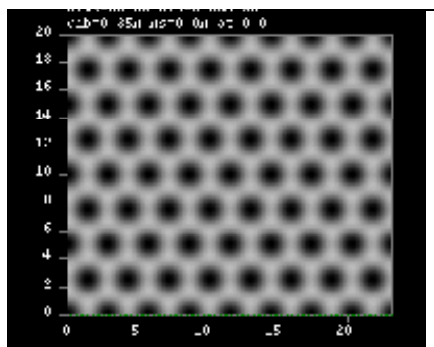

$\mathrm{t}=2.36 \AA$

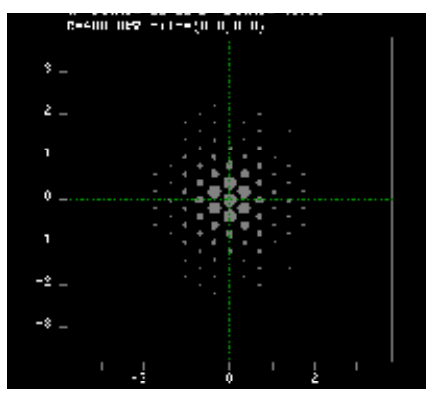

$3 a$

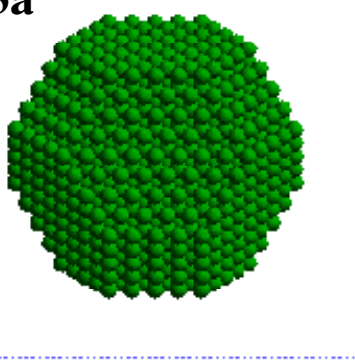

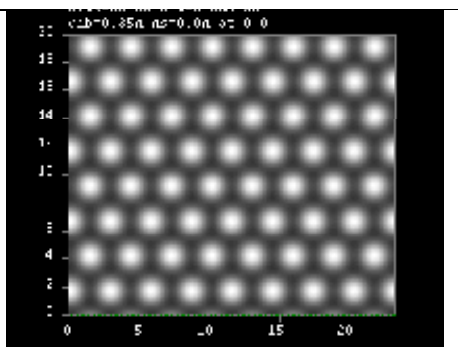

$\mathrm{t}=4.71 \AA$

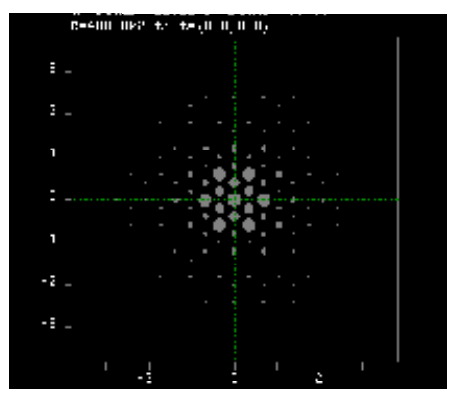

$3 b$

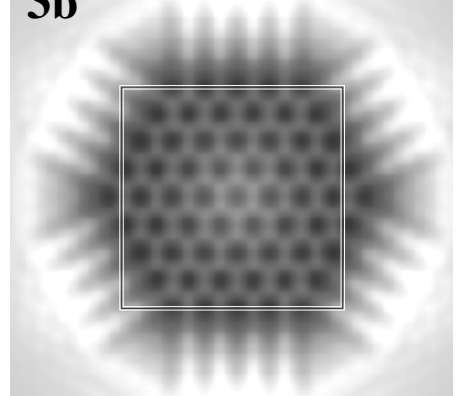

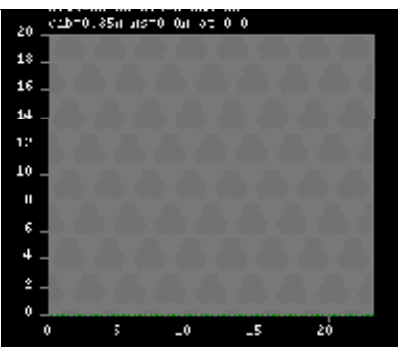

$\mathrm{t}=7.06 \AA$
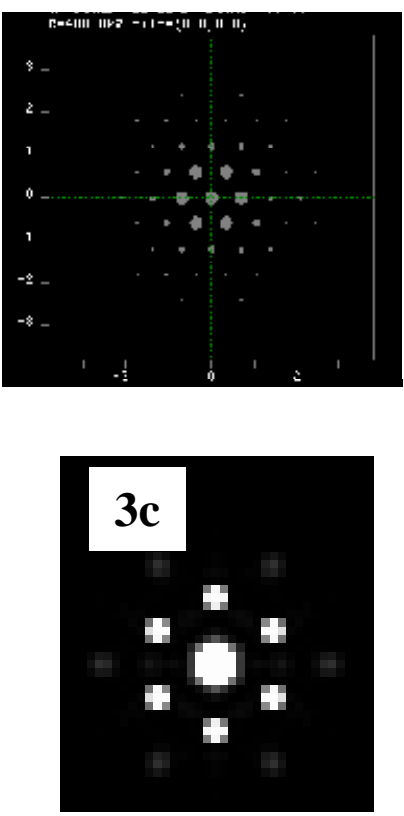

FIG. 1. (a),(b)Two experimental images of [111] Au particles and their diffractograms. FIG. 2. The simulated (a) images and (b) diffraction patterns for a Au thin film model. FIG. 3. (a) The top view of a 3-D projection of a cuboctahedron Au particle; (b) The simulated image at $-320 \AA$ and (c) its diffractogram . 